\title{
Protocol Author
}

National Cancer Institute

\section{Source}

National Cancer Institute. Protocol Author. NCI Thesaurus. Code C51853.

A person who is the writer of a structured research study. 